\title{
Correspondence
}

\section{Assessment of pediatric residents burnout in a tertiary academic center}

\section{To the Editor}

A recent study by Jamjoom and Parrk ${ }^{1}$ reported that $70 \%$ or more of pediatric residents experienced burnout and depersonalization, a sense of emotional distance from their patients or job. Notably, residents with spouses experienced more emotional exhaustion and depersonalization than singles, suggesting a relationship between burnout and related factors. Burnout was included in the International Classification of Diseases 10th revision (Diagnosis Code Z73.0), indicating it was recognized by the World Health Organization. ${ }^{2}$ Early identification and management of burnout should be emphasized in the training of health professionals because of the impact on physical, emotional, and psychological well-being. Korea has recently revised the law to strengthen the "human rights of the residents" in the "regulations on the training and qualification of medical specialists". ${ }^{3}$ Burnout varies across countries, social values, and cultural norms. As such, the results derived from the Jamjoom and Park study ${ }^{1}$ may not applicable to the Korean context. Nevertheless, this study highlights the need to protect the human rights of residents of our country. The protection of human rights, as it relates to the physical and mental health of residents, is important not only to the individual resident but also for the care and safety of patients. This endeavor indicates an awareness of social-cultural, educational, and political factors, and is in line with Korean goals of improving human rights and medical specialist training.

The causes of burnout can be broadly categorized into demographic characteristics, individual factors, and socio-cultural factors. Burnout varies according to demographic and personal characteristics ${ }^{4,5}$ and depends on the needs of organizational culture, sociocultural dimensions. In the present study, the prevalence of burnout in married residents was higher than in unmarried individuals. This outcome may be different from the outcome of previous studies suggesting that the presence of emotional supporters is associated with burnout. Additional studies, ${ }^{4,5}$ which examine the relationship between burnout and demographic characteristics and individual characteristics such as personality factors, (introversion and extroversion, instability) and degree of job satisfaction, can be useful in providing psychological counseling and developing guidance manual to prevent burnout among residents.
There are some limitations to this study. It seems that the number of study participants was very small to be able to reflect the situation of residents training program in Saudi. In particular, the sample size of PGY1 and PGY3 were especially small. There was a difference in the prevalence of burnout depending on the presence or absence of marriage; however, each group number is small. As shown in Table 1 and Table 2, the partner status distributions were different, and further explanation is needed. Moreover, there was no information about age. In Korea, there are various medical student selection systems, and male residents are required to provide military service. I am doubtful about the age of the residents surveyed in this study because the older the residents are, the more social experience they have and the more resistant they are to burnout. I would also like to know if there are any plans or suggestions to improve the residents training program in Saudi because of these issues.

Finally, this is a research which predicts the prevalence pattern and suggests solutions by recognizing the prevalence of burnout of the residents. If there is a research result that explores the factors of the burnout process, such as the process leading to burnout, the burnout phenomenon, how to cope with burnout, and the logical connection between these factors, it will help us to systemically understand the development and progression of burnout. In addition, it is important for other countries to explore ways to recognize and cope with the burnout of residents by considering burnout as a social, cultural, and national issue rather than as an individual issue.

Hyo H. Yoo
Department of Medical Education
Chonbuk National University School of Medicine Jeonju
Sang Y. Lee
Department of Medical Education
Pusan National University School of Medicine and
Family Medicine Clinic and Research Institute of
Convergence of Biomedical Science and Technology Pusan
National University Yangsan Hospital
Yangsan, Republic of Korea

\section{Reply from the Author}

Thank you Dr. Sang Lee for the interest in my work and the correspondence. As you know, burnout among physician, specially residents, is a serious worldwide issue affecting not only doctors' well-being but patient safety as well. This study is a pilot study at my academic 
center to explore the presence of the matter and its magnitude. That might explain the limited residents number. Since it is an assessment study to explore the dimension of the issue it did not include any root cause analysis. After the eye-opening results, we decided to dive deeper and find the root and how to mitigate this challenge through another qualitative study.

As for changing residency program to accommodate residents' needs and decrease burnout, this will be achievable as soon as we identify the causes and decide if it is program, institute or personal related. Nevertheless, we shared the alarming results with residency program director and the department. The plan is to involve the Saudi Commission of Health Specialties as soon as we complete the root cause analysis as it is the governing body of residency training in the whole country. I totally agree that burnout is an alarming phenomenon worldwide and we need to keep searching for other centers experiences and what solutions they came up with and worked.
Roaa S. Jamjoom Faculty of Medicine, Emergency Medicine Department, King Abdulaziz University,

References

Jeddah, Kingdom of Saudi Arabia

1. Jamjoom RS, Park YS. Assessment of pediatric residents burnout in a tertiary academic centre. Saudi Med J 2018; 39: 296-300.

2. Grossi G, Perski A, Osika W, Savic I. Stress-related exhaustion disorder--clinical manifestation of burnout? A review of assessment methods, sleep impairments, cognitive disturbances, and neuro-biological and physiological changes in clinical burnout. Scand J Psychol 2015; 56: 626-636.

3. Kim SS. Working conditions of interns/residents and patient safety: Painful training might not be authentic. J Korean Med Assoc 2016; 59: 82-84.

4. Sun JW, Lin PZ, Zhang HH, Li JH, Cao FL. A non-linear relationship between the cumulative exposure to occupational stressors and nurses' burnout and the potentially emotion regulation factors. J Ment Health 2017: 1-7.

5. Lacy BE, Chan JL. Physician burnout: the hidden health care crisis. Clin Gastroenterol Hepatol 2018; 16: 311-317. 